\title{
Controllability for a new class of fractional neutral integro-differential evolution equations with infinite delay and nonlocal conditions
}

\author{
Jun Du ${ }^{1,2^{*}}$, Wei Jiang ${ }^{1}$, Denghao Pang ${ }^{1}$ and Azmat Ullah Khan Niazi ${ }^{1}$
}

"Correspondence: djwlm@163.com 'School of Mathematical Science, Anhui University, Hefei, 230601،

People's Republic of China ${ }^{2}$ Department of Applied Mathematics, Huainan Normal University, Huainan, 232038, People's Republic of China

\begin{abstract}
In this paper, we apply the fractional calculus and a suitable fixed point theorem with the measure of noncompactness to give the sufficient conditions of the controllability for a new class of fractional neutral integro-differential evolution systems with infinite delay and nonlocal conditions. The results are obtained here under some weakly noncompactness conditions. Thus they improve and generalize many well-known results. At the end of this paper, two examples are given to explain our abstract conclusions.
\end{abstract}

MSC: 26A33; 34A08; 34A34; 93B05

Keywords: controllability; fractional integro-differential equations; neutral evolution equations; infinite delay; nonlocal conditions; fixed point theorem; measure of noncompactness

\section{Introduction}

In the last two decades, the theory of fractional differential equations have become an active area of investigation due to their applications in many fields such as viscoelasticity, electrochemistry, control, porous media, electromagnetic, etc. (see [1-3]). For more details of fractional calculus theory, one can see the monographs of Kilbas et al. [4], Miller and Ross [5], Podlubny [6], Baleanu [7]. In order to discuss the fractional systems in the abstract spaces, the first important step is how to define the new concept of a mild solution. A pioneering work has been reported by El-Borai [8] and Zhou and Jiao [9, 10]. Integrodifferential equations can be used to describe a lot of natural phenomena arising in many fields such as electronics, fluid dynamics, biological models and chemical kinetics. Most of these phenomena cannot be described through classical differential equations. That is why in recent years they have attracted more and more attention of many mathematicians, physicists, and engineers. Some topics for this kind of equations, such as existence and regularity, stability and control problems, have been investigated by many mathematicians; see [11-16] for example.

Recently, fractional calculus opened new perspectives in control theory. Many fundamental problems of control theory, such as pole assignment, stabilization and optimal

(c) The Author(s) 2017. This article is distributed under the terms of the Creative Commons Attribution 4.0 International License (http://creativecommons.org/licenses/by/4.0/), which permits unrestricted use, distribution, and reproduction in any medium, provided you give appropriate credit to the original author(s) and the source, provide a link to the Creative Commons license, and indicate if changes were made. 
control may be solved under the assumption that the system is controllable. The concept of controllability was firstly introduced by Kalman in 1960 and a systematic study was started after that. Most of the results in the existing literature are derived for finite dimensional systems. It should be pointed out that many unsolved problems still exist as far as controllability of infinite dimensional systems are concerned. In the case of infinite dimensional systems two basic concepts of controllability must be discriminated, which are exact and approximate controllability. Exact controllability enables one to steer the system to an arbitrary final state, while approximate controllability means that the system can be steered to an arbitrary small neighborhood of the final state. That is to say that exact controllability always implies approximate controllability. The converse statement is generally false. However, in the case of finite dimensional systems they coincide. There have been some results as regards the controllability of systems represented by nonlinear evolution equations in infinite dimensional spaces [14-24]. But when the semigroup is compact and other hypotheses are demanded, the application of exact controllability result is just restricted to the finite dimensional space [25]. As described in some papers, the nonlocal conditions may be connected with better effect in physical science than the classical initial conditions, since nonlocal conditions are normally more exact for physical estimations than the classical initial conditions. The study of abstract Cauchy problems with nonlocal initial conditions was initiated and proofs were given by Byszewski; see [26, 27]. Since the appearance of these two papers, several papers have addressed the issue of qualitative problems for various types of nonlinear differential equations with nonlocal conditions. We can refer to $[10,12,13,16-18,26]$. On the other hand, neutral differential equations with infinite delay arise in many areas of applied mathematics and for this reason these equations have received much attention in the last decades; see [11, 14, 20, 21, $28,29]$.

Very recently, Wang and Zhou [23] gave some conditions ensuring the complete controllability of fractional evolution systems without supposing the compactness of characteristic solution operators. Ravichandran and Baleanu [14] investigated the controllability of fractional functional integro-differential systems with an infinite delay in Banach spaces by means of fixed point theorem and phase space theory. Liang and Yang [16] presented weakly controllable conditions for the fractional evolution system with nonlocal initial conditions.

Inspired by these facts and $[9,14,16,23]$, in this manuscript we consider the controllability for a new class of fractional neutral integro-differential evolution systems with infinite delay and nonlocal initial conditions,

$$
\left\{\begin{array}{l}
{ }^{C} D^{q}\left[x(t)-g\left(t, x_{t}\right)\right]+A\left[x(t)-g\left(t, x_{t}\right)\right]=f\left(t, x_{t}, \Re x(t)\right)+B u(t), \quad t \in[0, a], \\
x(0)=\sum_{i=1}^{n} c_{i} x\left(t_{i}\right)+g\left(0, x_{0}\right), \quad x_{0}=\varphi \in \mathcal{B}_{l_{0}}, t \in(-\infty, 0],
\end{array}\right.
$$

where ${ }^{C} D^{q}$ is the Caputo fractional derivative of order $0<q<1, c_{i}(i=1, \ldots, n)$ are given constants and $0<t_{1}<t_{2}<\cdots<t_{n} \leq a . J=[0, a] .-A: D(A) \subset E \rightarrow E$ is the infinitesimal generator of a $C_{0}$-semigroup $T(t)(t \geq 0)$ of uniformly bounded linear operator in a Banach space $E$, for $T(t)(t \geq 0)$, there exists a constant $N \geq 1$ such that $\|T(t)\| \leq N$ for all $t \geq 0$. The control function $u$ is given in $L^{2}(J, U), U$ is a Banach spaces. $B$ is a linear bounded operator from $U$ to $E . g, f$ are given functions and satisfy some conditions that will be specified later. The time history $x_{t}:(-\infty, 0] \rightarrow E$ given by $x_{t}(\tau)=x(t+\tau)$ belongs to some abstract phase space $\mathcal{B}_{l_{0}}$ defined axiomatically. $\Re x(t)=\int_{0}^{t} \Upsilon(t, s) x(s) d s$, is a Volterra 
integral operator with integral kernel $\Upsilon \in C\left(\triangle, R^{+}\right), \Delta=\{(t, s): 0 \leq s \leq t \leq a\}$. We always suppose that $\Upsilon^{*}=\sup _{t \in J} \int_{0}^{t} \Upsilon(t, s) d s$.

By using a concrete type of nonlocal function in our present manuscript, we eliminate the compactness of nonlocal function, only suppose that $c_{i}(i=1,2, \ldots, n)$ satisfy the condition (H0) (see Section 2). And we omit the assumptions for compactness of the $C_{0}$ semigroup $T(t)(t \geq 0)$. Furthermore, we concentrate on a new class of neutral nonlocal control systems with infinite delay and establish sufficient conditions for the controllability of the system (1) by relying on a measure of noncompactness and the Mönch fixed point theorem in addition to new phase space axioms. When $g\left(t, x_{t}\right) \equiv 0$ and $\tau=0$, then system (1) is degenerated to the case of [16].

The rest of this work is arranged as follows. In Section 2, some notations and preparation results are presented. In Section 3, by the Mönch fixed point theorem, we prove the exact controllability of fractional neutral integro-differential evolution equations with nonlocal conditions and infinite delay. In Section 4, two examples are given to explain our abstract conclusions.

\section{Preliminaries and lemmas}

In this section, we mention notations, definitions, lemmas and preliminary facts needed to obtain our main results.

We assume that $(E,\|\cdot\|)$ is a Banach space. Denote $C(J, E)$ for the Banach space of continuous functions from $J$ into $E$ with the norm $\|x\|=\sup _{t \in J}|x(t)|, x \in C(J, E) . L^{p}(J, E)$ $(1 \leq p<\infty)$ denotes the Banach space of measurable functions $x: J \rightarrow E$ which are Bochner integrable normed by $\|x\|_{p}=\left(\int_{0}^{a}\|x(t)\|^{p} d t\right)^{\frac{1}{p}}, x \in L^{p}(J, E)$.

We now define the phase space $\mathcal{B}_{l_{0}}$. Assume that $l:(-\infty, 0] \rightarrow(0,+\infty)$ is a continuous function with $l_{0}=\int_{-\infty}^{0} l(t) d t<+\infty$. The Banach space $\left(\mathcal{B}_{l_{0}},\|\cdot\|_{\mathcal{B}_{l_{0}}}\right)$ induced by the function $l(t)$ is defined as follows.

$$
\begin{aligned}
\mathcal{B}_{l_{0}}= & \{\varphi:(-\infty, 0] \rightarrow E: \varphi \text { is a bounded and measurable function on }[-\delta, 0] \\
& \text { and } \left.\int_{-\infty}^{0} l(t) \sup _{t \leq \tau \leq 0}|\varphi(\tau)| d t<+\infty\right\}
\end{aligned}
$$

endowed with the norm $\|\varphi\|_{\mathcal{B}_{l_{0}}}:=\int_{-\infty}^{0} l(t) \sup _{t \leq \tau \leq 0}|\varphi(\tau)| d t$.

Now we consider the space

$$
\begin{aligned}
\mathcal{B}_{l_{a}}= & \left\{x:(-\infty, a] \rightarrow E:\left.x\right|_{J} \in C(J, E) \text {, and } x_{0}=\varphi \in \mathcal{B}_{l_{0}}\right. \\
& \text { such that } \left.x(0)=\sum_{i=0}^{n} c_{i} x\left(t_{i}\right)+g\left(0, x_{0}\right)\right\} .
\end{aligned}
$$

Let $\|\cdot\|_{a}$ be a seminorm in the space $\mathcal{B}_{l_{a}}$ defined by

$$
\|x\|_{a}=\|\varphi\|_{\mathcal{B}_{l_{0}}}+\sup \{\|x(t)\|: t \in[0, a]\}, \quad x \in \mathcal{B}_{l_{a}} .
$$

Lemma $2.1([20,21])$ Assume $x \in \mathcal{B}_{l_{a}}$, then, for $t \in J, x_{t} \in \mathcal{B}_{l_{0}}$. Moreover,

$$
l_{0}|x(t)| \leq\left\|x_{t}\right\|_{\mathcal{B}_{l_{0}}} \leq\|\varphi\|_{\mathcal{B}_{l_{0}}}+l_{0} \sup _{s \in[0, t]}|x(s)|
$$


Definition 2.1 ([6]) The fractional integral of order $\gamma$ with the lower limit 0 for a function $f$ is written as

$$
I^{\gamma} f(t)=\frac{1}{\Gamma(\gamma)} \int_{0}^{t} \frac{f(s)}{(t-s)^{1-\gamma}} d s, \quad t>0, \gamma>0
$$

provided the right-hand side is pointwise defined on $[0, \infty)$, where $\Gamma$ is the gamma function.

Definition 2.2 ([6]) Riemann-Liouville derivative of order $\gamma$ with the lower limit 0 for a function $f:[0, \infty) \rightarrow R$ can be defined as

$$
{ }^{R-L} D^{\gamma} f(t)=\frac{1}{\Gamma(n-\gamma)} \frac{d^{n}}{d t^{n}} \int_{0}^{t} \frac{f(s)}{(t-s)^{\gamma+1-n}} d s, \quad t>0,0 \leq n-1<\gamma<n .
$$

Definition 2.3 ([6]) The Caputo derivative of order $\gamma$ for a function $f:[0, \infty) \rightarrow R$ can be denoted by

$$
{ }^{C} D^{\gamma} f(t)={ }^{R-L} D^{\gamma}\left(f(t)-\sum_{k=0}^{n-1} \frac{t^{k}}{k !} f^{(k)}(0)\right), \quad t>0,0 \leq n-1<\gamma<n .
$$

\section{Remark 2.1}

(i) If $f(t) \in C^{n}[0, \infty)$ then

$$
{ }^{C} D^{\gamma} f(t)=\frac{1}{\Gamma(n-\gamma)} \int_{0}^{t} \frac{f^{(n)}(s)}{(t-s)^{\gamma+1-n}} d s=I^{n-\gamma} f^{(n)}(t), \quad t>0,0 \leq n-1<\gamma<n
$$

(ii) the Caputo derivative of a constant is equal to zero;

(iii) if $f$ is an abstract function with values in $E$, then the integrals which are presented in Definitions 2.1 and 2.2 are taken in Bochner's sense.

For any $x \in E$, define two operators $\{\varpi(t)\}_{t \geq 0}$ and $\{v(t)\}_{t \geq 0}$ by

$$
\begin{aligned}
& \varpi(t) x=\int_{0}^{\infty} \pi_{q}(\vartheta) T\left(t^{q} \vartheta\right) x d \vartheta, \\
& v(t) x=q \int_{0}^{\infty} \vartheta \pi_{q}(\vartheta) T\left(t^{q} \vartheta\right) x d \vartheta, \quad 0<q<1,
\end{aligned}
$$

where

$$
\begin{aligned}
& \pi_{q}(\vartheta)=\frac{1}{q} \vartheta^{-1-\frac{1}{q}} \varrho_{q}\left(\vartheta^{-\frac{1}{q}}\right), \\
& \varrho_{q}(\vartheta)=\frac{1}{\pi} \sum_{n=1}^{\infty}(-1)^{n-1} \vartheta^{-q n-1} \frac{\Gamma(n q+1)}{n !} \sin (n \pi q), \quad \vartheta \in(0, \infty) .
\end{aligned}
$$

$\pi_{q}$ is a probability density function defined on $(0, \infty)$, which satisfies $\pi_{q}(\vartheta) \geq 0$ for all $\vartheta \in(0, \infty)$ and $\int_{0}^{\infty} \pi_{q}(\vartheta) d \vartheta=1$. Moreover, the operators $\{\varpi(t)\}_{t \geq 0}$ and $\{v(t)\}_{t \geq 0}$ have the following properties. 
Lemma 2.2 ([9]) The operators $\varpi(t)$ and $v(t)$ satisfy:

(i) For any fixed $t \geq 0$ and any $x \in E$, the following inequalities hold.

$$
\|\varpi(t) x\| \leq N\|x\|, \quad\|v(t) x\| \leq \frac{N}{\Gamma(q)}\|x\| .
$$

(ii) The operators $\varpi(t)$ and $v(t)$ are strongly continuous for all $t \geq 0$.

(iii) If $T(t)(t \geq 0)$ is an equicontinuous semigroup, then $\varpi(t)$ and $v(t)$ are equicontinuous in $E$ for $t>0$.

Definition 2.4 ([30]) Let $E$ be a Banach space and $\Omega_{E} \subset E$ be bounded. The Hausdorff measure of noncompactness is the map $\chi: \Omega_{E} \rightarrow[0, \infty)$ defined by

$\chi(\Lambda)=\inf \left\{\epsilon>0: \Lambda\right.$ has a finite $\epsilon$-net in $\left.\Omega_{E}\right\}$.

We need to use the following basic properties of MNC $\chi$; see [17, 31]. For all bounded subsets $\Lambda, \Lambda_{1}, \Lambda_{2}$ of $E$, we have

(i) $\Lambda_{1} \subset \Lambda_{2} \Rightarrow \chi\left(\Lambda_{1}\right) \leq \chi\left(\Lambda_{2}\right)$;

(ii) $\chi\left(\Lambda_{1}+\Lambda_{2}\right) \leq \chi\left(\Lambda_{1}\right)+\chi\left(\Lambda_{2}\right)$, where $\Lambda_{1}+\Lambda_{2}=\left\{x+y: x \in \Lambda_{1}, y \in \Lambda_{2}\right\}$;

(iii) $\chi\left(\Lambda_{1} \cup \Lambda_{2}\right) \leq \max \left\{\chi\left(\Lambda_{1}\right), \chi\left(\Lambda_{2}\right)\right\}$;

(iv) $\chi(\sigma \Lambda) \leq|\sigma| \chi(\Lambda)$ for any $\sigma \in R$;

(v) $\chi(\{e\} \cup \Lambda)=\chi(\Lambda)$ for any $e \in E$;

(vi) $\chi(\Lambda)=0 \Leftrightarrow \Lambda$ is relatively compact in $E$.

Lemma 2.3 ([30]) For any $G \subset C(J, E)$ and $t \in J$, define $G(t)=\{u(t) \in E: u \in G\}$. If $G$ is bounded and equicontinuous, then $\chi(G(t))$ is continuous on $J$ and $\chi(G)=\max _{t \in J} \chi(G(t))$.

Lemma 2.4 ([32]) Let $\left\{\varphi_{n}\right\}_{n=1}^{\infty}$ be a sequence of Bochner integrable functions from J into $E$. If there exists $\varphi \in L^{1}\left(J, R^{+}\right)$such that $\left\|\varphi_{n}(t)\right\| \leq \varphi(t)$ a.e. $t \in J, n=1,2, \ldots$, then $G(t)=$ $\chi\left(\left\{\varphi_{n}(t)\right\}_{n=1}^{\infty}\right)$ belongs to $L^{1}\left(J, R^{+}\right)$and satisfies

$$
\chi\left(\left\{\int_{J} \varphi_{n}(t) d t: n \in N\right\}\right) \leq 2 \int_{J} \chi(G(t)) d t .
$$

Lemma 2.5 ([9]) Assume that $p_{1}, p_{2} \geq 1$, and $\frac{1}{p_{1}}+\frac{1}{p_{2}}=1$. If $m_{1} \in L^{p_{1}}(J, R), m_{2} \in L^{p_{2}}(J, R)$, then, for $m_{1} m_{2} \in L^{1}(J, R)$, one has

$$
\left\|m_{1} m_{2}\right\|_{L^{1 J}} \leq\left\|m_{1}\right\|_{L^{p_{1} J}}\left\|m_{2}\right\|_{L^{p_{2} J}} .
$$

Lemma 2.6 ([33]) Let $D$ be a convex, closed set in a Banach space $E$ with $0 \in D$. Suppose there is a continuous map $\Psi: D \rightarrow D$ with the following property: for $W \subset D$ is countable and $W \subset \overline{c o}(\{0\} \cup \Psi(W))$ imply that $W$ is relatively compact. Then $\Psi$ has at least a fixed point in $D$.

\section{Controllability results}

First, we discuss the following neutral evolution equation with nonlocal conditions:

$$
\left\{\begin{array}{l}
{ }^{C} D^{q}\left[x(t)-g\left(t, x_{t}\right)\right]+A\left[x(t)-g\left(t, x_{t}\right)\right]=h(t), \quad t \in J, \\
x(0)=\sum_{i=1}^{n} c_{i} x\left(t_{i}\right)+g\left(0, x_{0}\right), \quad x_{0}=\varphi \in \mathcal{B}_{l_{0}}, t \in(-\infty, 0],
\end{array}\right.
$$

where $h \in C((-\infty, a], E)$. 
According to Definitions 2.1, 2.2 and 2.3, it is appropriate to change the system (2) into the equivalent integral equation

$$
\begin{aligned}
x(t)= & x(0)-g\left(0, x_{0}\right)+g\left(t, x_{t}\right) \\
& +\frac{1}{\Gamma(q)} \int_{0}^{t}(t-s)^{q-1}\left\{-A\left[x(s)-g\left(s, x_{s}\right)\right]+h(s)\right\} d s, \quad t \in J,
\end{aligned}
$$

provided that the integral in (3) exists.

Before giving the definition of mild solution of the system (1), we first prove the following lemmas.

Lemma 3.1 If the integral equation (3) holds, then we have

$$
x(t)=\varpi(t)\left[x(0)-g\left(0, x_{0}\right)\right]+g\left(t, x_{t}\right)+\int_{0}^{t}(t-s)^{q-1} v(t-s) h(s) d s, \quad t \in J,
$$

where $\varpi$ and $v$ are defined as previously.

Proof Let $\lambda>0$. Applying the Laplace transform

$$
S(\lambda)=\int_{0}^{\infty} e^{-\lambda s} x(s) d s, \quad Y(\lambda)=\int_{0}^{\infty} e^{-\lambda s} g\left(s, x_{s}\right) d s, \quad Z(\lambda)=\int_{0}^{\infty} e^{-\lambda s} h(s) d s
$$

to (3), we get

$$
\begin{aligned}
S(\lambda)= & \frac{1}{\lambda}\left[x(0)-g\left(0, x_{0}\right)\right]+Y(\lambda)-\frac{1}{\lambda q} A S(\lambda)+\frac{1}{\lambda^{q}} A Y(\lambda)+\frac{1}{\lambda^{q}} Z(\lambda) \\
= & \lambda^{q-1}\left(\lambda^{q} I+A\right)^{-1}\left[x(0)-g\left(0, x_{0}\right)\right]+Y(\lambda)+\left(\lambda^{q} I+A\right)^{-1} Z(\lambda) \\
= & \lambda^{q-1} \int_{0}^{\infty} e^{-\lambda^{q} s} T(s)\left[x(0)-g\left(0, x_{0}\right)\right] d s+\int_{0}^{\infty} e^{-\lambda s} g\left(s, x_{s}\right) d s \\
& +\int_{0}^{\infty} e^{-\lambda^{q} s} T(s) Z(\lambda) d s,
\end{aligned}
$$

provided that the integral (4) exists, where $I$ is the identity operator defined on $E$.

Let

$$
\varrho_{q}(\vartheta)=\frac{q}{\vartheta^{q+1}} \pi_{q}\left(\vartheta^{-q}\right)
$$

whose Laplace transform is given by

$$
\int_{0}^{\infty} e^{-\lambda \vartheta} \varrho_{q}(\vartheta) d \vartheta=e^{-\lambda^{q}}, \quad q \in(0,1)
$$

Using (5), we have

$$
\begin{aligned}
& \lambda^{q-1} \int_{0}^{\infty} e^{-\lambda^{q} s} T(s)\left[x(0)-g\left(0, x_{0}\right)\right] d s \\
& \quad=\int_{0}^{\infty} q(\lambda t)^{q-1} e^{-(\lambda t)^{q}} T\left(t^{q}\right)\left[x(0)-g\left(0, x_{0}\right)\right] d t
\end{aligned}
$$




$$
\begin{aligned}
& =\int_{0}^{\infty}-\frac{1}{\lambda} \frac{d}{d t}\left[e^{-(\lambda t)^{q}}\right] T\left(t^{q}\right)\left[x(0)-g\left(0, x_{0}\right)\right] d t \\
& =\int_{0}^{\infty} \int_{0}^{\infty} \vartheta \varrho_{q}(\vartheta) e^{-\lambda t \vartheta} T\left(t^{q}\right)\left[x(0)-g\left(0, x_{0}\right)\right] d \vartheta d t \\
& =\int_{0}^{\infty} e^{-\lambda t}\left\{\int_{0}^{\infty} \varrho_{q}(\vartheta) T\left(\frac{t^{q}}{\vartheta q}\right)\left[x(0)-g\left(0, x_{0}\right)\right] d \vartheta\right\} d t \\
& =\int_{0}^{\infty} e^{-\lambda t} \int_{0}^{\infty} \pi_{q}(\vartheta) T\left(t^{q} \vartheta\right)\left[x(0)-g\left(0, x_{0}\right)\right] d \vartheta d t
\end{aligned}
$$

and

$$
\begin{aligned}
\int_{0}^{\infty} & e^{-\lambda q_{s}} T(s) Z(\lambda) d s \\
& =\int_{0}^{\infty} \int_{0}^{\infty} q t^{q-1} e^{-(\lambda t)^{q}} T\left(t^{q}\right) e^{-\lambda s} h(s) d s \\
& =\int_{0}^{\infty} \int_{0}^{\infty} \int_{0}^{\infty} q \varrho_{q}(\vartheta) e^{-(\lambda t \vartheta)} T\left(t^{q}\right) e^{-\lambda s} t^{q-1} h(s) d \vartheta d s d t \\
& =\int_{0}^{\infty} \int_{0}^{\infty} \int_{0}^{\infty} q \varrho_{q}(\vartheta) e^{-\lambda(t+s)} T\left(\frac{t^{q}}{\vartheta q}\right) \frac{t^{q-1}}{\vartheta q} h(s) d \vartheta d s d t \\
& =\int_{0}^{\infty} e^{-\lambda t}\left[q \int_{0}^{t} \int_{0}^{\infty} \varrho_{q}(\vartheta) T\left(\frac{(t-s)^{q}}{\vartheta^{q}}\right) \frac{(t-s)^{q}}{\vartheta q} h(s) d \vartheta d s\right] d t \\
& =\int_{0}^{\infty} e^{-\lambda t}\left[q \int_{0}^{t} \int_{0}^{\infty} \vartheta(t-s)^{q-1} \pi_{q}(\vartheta) T\left((t-s)^{q} \vartheta\right) h(s) d \vartheta d s\right] d t
\end{aligned}
$$

According to (4), (7), (8) and using the Laplace inverse transform, we obtain

$$
x(t)=\varpi(t)\left[x(0)-g\left(0, x_{0}\right)\right]+g\left(t, x_{t}\right)+\int_{0}^{t}(t-s)^{q-1} v(t-s) h(s) d s, \quad t \in J .
$$

This completes the proof.

Suppose that there exists the bounded operator $K: E \rightarrow E$ given by

$$
K:=\left[I-\sum_{i=1}^{n} c_{i} \varpi\left(t_{i}\right)\right]^{-1}
$$

By means of [34] we can present the sufficient conditions for the existence and boundedness of the operator $K$.

Lemma 3.2 The operator $K$ defined in (9) exists and is bounded if the following condition holds:

(H0) there are real numbers $c_{i}$ such that

$$
\sum_{i=1}^{n}\left|c_{i}\right|<\frac{1}{N}
$$


Proof From the hypothesis (H0), we have

$$
\left\|\sum_{i=1}^{n} c_{i} \varpi\left(t_{i}\right)\right\| \leq \sum_{i=1}^{n}\left|c_{i}\right| \cdot\left\|\varpi\left(t_{i}\right)\right\|<1 .
$$

By the operator spectrum theorem, the operator $K=\left[I-\sum_{i=1}^{n} c_{i} \varpi\left(t_{i}\right)\right]^{-1}$ exists and is bounded. In addition, by the Neumann expression, we get

$$
\|K\| \leq \sum_{n=0}^{\infty}\left\|\sum_{i=1}^{n} c_{i} \varpi\left(t_{i}\right)\right\|^{n}=\frac{1}{1-\left\|\sum_{i=1}^{n} c_{i} \varpi\left(t_{i}\right)\right\|} \leq \frac{1}{1-N \sum_{i=1}^{n}\left|c_{i}\right|}
$$

Using Lemmas 3.1, 3.2, we give the following definition of a mild solution of the neutral system (2) with nonlocal conditions.

Definition 3.1 A function $x:(-\infty, a] \rightarrow E$ satisfies the conditions:

(i)

$$
x(t)=\sum_{i=1}^{n} c_{i} \varpi(t) K g\left(t_{i}, x_{t_{i}}\right)+g\left(t, x_{t}\right)+\sum_{i=1}^{n} c_{i} \varpi(t) K H\left(t_{i}\right)+H(t), \quad t \in J,
$$

where $K=\left[I-\sum_{i=1}^{n} c_{i} \varpi\left(t_{i}\right)\right]^{-1} ; H(t)=\int_{0}^{t}(t-s)^{q-1} v(t-s) h(s) d s$;

(ii) $x_{0}=\varphi(t) \in \mathcal{B}_{l_{0}}$ s.t. $x(0)=\sum_{i=1}^{n} c_{i} x\left(t_{i}\right)+g\left(0, x_{0}\right), t \in(-\infty, 0]$.

This is called a mild solution of the nonlocal Cauchy problem (2).

Remark 3.1 Due to Lemma 3.1, a mild solution to fractional evolution equation (2) with the initial condition is

$$
x(t)=\varpi(t)\left[x(0)-g\left(0, x_{0}\right)\right]+g\left(t, x_{t}\right)+\int_{0}^{t}(t-s)^{q-1} v(t-s) h(s) d s, \quad t \in J .
$$

Specially,

$$
x\left(t_{i}\right)=\varpi\left(t_{i}\right) x(0)-\varpi\left(t_{i}\right) g\left(0, x_{0}\right)+g\left(t_{i}, x_{t_{i}}\right)+\int_{0}^{t_{i}}\left(t_{i}-s\right)^{q-1} v\left(t_{i}-s\right) h(s) d s .
$$

Using (2) and (13), we get

$$
\begin{aligned}
x(0)-g\left(0, x_{0}\right)= & \sum_{i=1}^{n} c_{i} \varpi\left(t_{i}\right) x(0)-\sum_{i=1}^{n} c_{i} \varpi\left(t_{i}\right) g\left(0, x_{0}\right)+\sum_{i=1}^{n} c_{i} g\left(t_{i}, x_{t_{i}}\right) \\
& +\sum_{i=1}^{n} c_{i} \int_{0}^{t_{i}}\left(t_{i}-s\right)^{q-1} v\left(t_{i}-s\right) h(s) d s .
\end{aligned}
$$

Since $I-\sum_{i=1}^{n} c_{i} \varpi\left(t_{i}\right)$ exists, there exists a bounded inverse operator which is denoted by $K$, so that $x(0)=g\left(0, x_{0}\right)+\sum_{i=1}^{n} c_{i} K g\left(t_{i}, x_{t_{i}}\right)+\sum_{i=1}^{n} c_{i} K \int_{0}^{t_{i}}\left(t_{i}-s\right)^{q-1} v\left(t_{i}-s\right) h(s) d s$. And hence

$$
x(t)=\sum_{i=1}^{n} c_{i} \varpi(t) K g\left(t_{i}, x_{t_{i}}\right)+g\left(t, x_{t}\right)+\sum_{i=1}^{n} c_{i} \varpi(t) K H\left(t_{i}\right)+H(t), \quad t \in J,
$$

it is exactly (12). 
Similarly, we present the following definition.

Definition 3.2 A function $x:(-\infty, a] \rightarrow E$ is called a mild solution of the nonlocal control system (1), if $x_{0}=\varphi \in \mathcal{B}_{l_{0}}$ s.t. $x(0)=\sum_{i=1}^{n} c_{i} x\left(t_{i}\right)+g\left(0, x_{0}\right)$, and, for any $u \in L^{2}(J, U)$, the integral equation

$$
\begin{aligned}
x(t)= & \sum_{i=1}^{n} c_{i} \varpi(t) K \int_{0}^{t_{i}}\left(t_{i}-s\right)^{q-1} v\left(t_{i}-s\right)\left[B u(s)+f\left(s, x_{s}, \Re x(s)\right)\right] d s \\
& +\sum_{i=1}^{n} c_{i} \varpi(t) K g\left(t_{i}, x_{t_{i}}\right)+\int_{0}^{t}(t-s)^{q-1} v(t-s)\left[B u(s)+f\left(s, x_{s}, \Re x(s)\right)\right] d s \\
& +g\left(t, x_{t}\right), \quad t \in J,
\end{aligned}
$$

is satisfied.

To present and prove the main results of this paper, we list the following hypotheses:

(H1) $-A$ generates an equicontinuous semigroup $T(t)(t \geq 0)$ of uniformly bounded linear operators in $E$.

(H2) (1) The linear operator $\Im: L^{2}(J, U) \rightarrow E$ defined by

$$
\begin{aligned}
\Im u= & \varpi(a) \sum_{i=1}^{n} c_{i} K \int_{0}^{t_{i}}\left(t_{i}-s\right)^{q-1} v\left(t_{i}-s\right) B u(s) d s \\
& +\int_{0}^{a}(a-s)^{q-1} v(a-s) B u(s) d s
\end{aligned}
$$

is reversible, the inverse operator is denoted by $\mathfrak{I}^{-1}$ and takes values in

$L^{2}(J, U) \operatorname{ker} \Im$, and there exist two constants $N_{1}>0, N_{2}>0$ such that $\|B\| \leq N_{1}$, $\left\|\Im^{-1}\right\| \leq N_{2}$

(2) there exist a constant $q_{1} \in(0, q)$ and $\xi_{1} \in L^{\frac{1}{q_{1}}}\left(J, R^{+}\right)$such that

$$
\chi\left(\Im^{-1}(W)(t)\right) \leq \xi_{1}(t) \chi(W), \quad t \in J,
$$

for any countable subset $W \subset E$.

(H3) The function $f: J \times \mathcal{B}_{l_{0}} \times E \rightarrow E$ satisfies:

(1) The function $f(t, \cdot, \cdot)$ is continuous for each $t \in J$, and the function $f(\cdot, \varphi, x)$ is strongly measurable for any $(\varphi, x) \in \mathcal{B}_{l_{0}} \times E$;

(2) for any countable sets $V_{1} \subset \mathcal{B}_{l_{0}}, W_{1} \subset E$, there exist a constant $q_{2} \in(0, q)$ and $\xi_{2} \in L^{\frac{1}{q_{2}}}\left(J, R^{+}\right)$such that

$$
\chi\left(f\left(t, V_{1}, W_{1}\right)\right) \leq \xi_{2}(t)\left(\sup _{-\infty<\tau \leq 0} \chi\left(V_{1}(\tau)\right)+\chi\left(W_{1}\right)\right), \quad t \in J ;
$$

(3) for any $r>0$, there exist a constant $q_{3} \in(0, q)$ and $S_{r} \in L^{\frac{1}{q_{3}}}\left(J, R^{+}\right)$such that, for any $(\varphi, y) \in \mathcal{B}_{l_{0}} \times E$,

$$
\sup \left\{\|f(t, \varphi, y)\|:\|\varphi\|_{\mathcal{B}_{l_{0}}} \leq r^{\prime},\|y\| \leq \Upsilon^{*} r\right\} \leq S_{r}(t), \quad t \in J
$$

where $S_{r}$ satisfies $\liminf _{r \rightarrow+\infty} \frac{1}{r}\left\|S_{r}\right\|_{L^{\frac{1}{q 3}}}=\gamma<\infty$. 
(H4) (1) The function $g: J \times \mathcal{B}_{l_{0}} \rightarrow E$ is continuous, there exists $x_{0}=\varphi$ s.t. $x(0)=\sum_{i=1}^{n} c_{i} x\left(t_{i}\right)+g\left(0, x_{0}\right), t \in(-\infty, 0]$ and there exist nonnegative constants $H_{1}, H_{2}, H_{3}, 0<\beta<1$ such that, for any $t \in J, z, y \in \mathcal{B}_{l_{0}}, g(\cdot, \cdot)$ satisfies the inequality

$$
\|g(t, z)\| \leq H_{1}\left(1+\|z\|_{\mathcal{B}_{l_{0}}}\right)
$$

and the Lipschitz condition

$$
\left\|g\left(t_{1}, z\right)-g\left(t_{2}, y\right)\right\| \leq H_{2}\|z-y\|_{\mathcal{B}_{l_{0}}}+H_{3}\left|t_{1}-t_{2}\right|
$$

(2) for any countable subset $W_{2} \subset \mathcal{B}_{l_{0}}$, there exists a nonnegative bounded function $\xi_{3}$ such that

$$
\chi\left(g\left(t, W_{2}\right)\right) \leq \xi_{3}(t) \sup _{-\infty<\tau \leq 0} \chi\left(W_{2}(\tau)\right), \quad t \in J
$$

where $\sup _{t \in[0, a]} \xi_{3}(t)=\kappa$.

By the hypothesis $(\mathrm{H} 2)(1)$, for any $x_{1} \in E$, we define a feedback control function $u(t):=$ $u(t ; x)$ as follows:

$$
\begin{aligned}
u(t ; x)= & \Im^{-1}\left[x_{1}-\varpi(a) \sum_{i=1}^{n} c_{i} K \int_{0}^{t_{i}}\left(t_{i}-s\right)^{q-1} v\left(t_{i}-s\right) f\left(s, x_{s}, \Re x(s)\right) d s-g\left(a, x_{a}\right)\right. \\
& \left.-\varpi(a) \sum_{i=1}^{n} c_{i} K g\left(t_{i}, x_{t_{i}}\right)-\int_{0}^{a}(a-s)^{q-1} v(a-s) f\left(s, x_{s}, \Re x(s)\right) d s\right](t), \quad t \in J .
\end{aligned}
$$

For convenience, let us take the following notations:

$$
\mathcal{P}(t ; x)=B u(t ; x)+f\left(t, x_{t}, \Re x(t)\right) ; \quad \tilde{\mathcal{P}}(x)=\sum_{i=1}^{n} c_{i} K \int_{0}^{t_{i}}\left(t_{i}-s\right)^{q-1} v\left(t_{i}-s\right) \mathcal{P}(s ; x) d s
$$

and

$$
d_{i}=\frac{a^{q-q_{i}}}{\left(b_{i}+1\right)^{1-q_{i}}}, \quad b_{i}=\frac{q-1}{1-q_{i}}, \quad i=1,2,3 .
$$

We consider the operator $\Psi: \mathcal{B}_{l_{a}} \rightarrow \mathcal{B}_{l_{a}}$ defined by

$$
(\Psi x)(t)=\left\{\begin{array}{rlrl}
\varphi(t), & t \in(-\infty, 0], \\
\sum_{i=1}^{n} c_{i} \varpi(t) K g\left(t_{i}, x_{t_{i}}\right)+g\left(t, x_{t}\right) & \\
& +\sum_{i=1}^{n} c_{i} \varpi(t) K \int_{0}^{t_{i}}\left(t_{i}-s\right)^{q-1} v\left(t_{i}-s\right) B u(s) d s & \\
& +\int_{0}^{t}(t-s)^{q-1} v(t-s) B u(s) d s & \\
& +\sum_{i=1}^{n} c_{i} \varpi(t) K \int_{0}^{t_{i}}\left(t_{i}-s\right)^{q-1} v\left(t_{i}-s\right) f\left(s, x_{s}, \Re x(s)\right) d s & \\
& +\int_{0}^{t}(t-s)^{q-1} v(t-s) f\left(s, x_{s}, \Re x(s)\right) d s, & t \in J,
\end{array}\right.
$$

where $x_{0}=\varphi \in \mathcal{B}_{l_{0}}$ satisfying $x(0)=\sum_{i=1}^{n} c_{i} x\left(t_{i}\right)+g(0, \varphi)$. 
For $\varphi \in \mathcal{B}_{l_{0}}$, we define $\tilde{\varphi}$ by

$$
\tilde{\varphi}(t)= \begin{cases}\varphi(t), & t \in(-\infty, 0], \\ 0, & t \in J,\end{cases}
$$

then $\tilde{\varphi} \in \mathcal{B}_{l_{a}}$. Set $x(t)=z(t)+\tilde{\varphi}(t),-\infty<t \leq a$. It is easy to see that $x$ satisfies $z_{0}=0$, $t \in(-\infty, 0]$ and

$$
\begin{aligned}
z(t)= & \sum_{i=1}^{n} c_{i} \varpi(t) K g\left(t_{i}, z_{t_{i}}+\tilde{\varphi}_{t_{i}}\right)+g\left(t, z_{t}+\tilde{\varphi}_{t}\right) \\
& +\sum_{i=1}^{n} c_{i} \varpi(t) K \int_{0}^{t_{i}}\left(t_{i}-s\right)^{q-1} v\left(t_{i}-s\right) f\left(s, z_{s}+\tilde{\varphi}_{s}, \Re x(s)\right) d s \\
& +\int_{0}^{t}(t-s)^{q-1} v(t-s) f\left(s, z_{s}+\tilde{\varphi}_{s}, \Re x(s)\right) d s \\
& +\sum_{i=1}^{n} c_{i} \varpi(t) K \int_{0}^{t_{i}}\left(t_{i}-s\right)^{q-1} v\left(t_{i}-s\right) B u(s) d s \\
& +\int_{0}^{t}(t-s)^{q-1} v(t-s) B u(s) d s .
\end{aligned}
$$

Let $\mathcal{B}_{l_{a}}^{0}=\left\{z \in \mathcal{B}_{l_{a}}: z_{0}=0 \in \mathcal{B}_{l_{0}}\right\}$. For any $z \in \mathcal{B}_{l_{a}}^{0}$

$$
\begin{aligned}
\|z\|_{a} & =\left\|z_{0}\right\|_{\mathcal{B}_{l_{0}}}+\sup \{\|z(t)\|: 0 \leq t \leq a\} \\
& =\sup \{\|z(t)\|: 0 \leq t \leq a\},
\end{aligned}
$$

thus $\left(\mathcal{B}_{l_{a}}^{0},\|\cdot\|_{a}\right)$ is a Banach space. Set $B_{r}=\left\{z \in \mathcal{B}_{l_{a}}^{0}:\|z\|_{a} \leq r\right\}$ for some $r>0$, then $B_{r} \subseteq \mathcal{B}_{l_{a}}^{0}$ is uniformly bounded, and for $z \in B_{r}$, from Lemma 2.1, we have

$$
\left\|z_{t}+\tilde{\varphi}_{t}\right\|_{\mathcal{B}_{l_{0}}} \leq\left\|z_{t}\right\|_{\mathcal{B}_{l_{0}}}+\left\|\tilde{\varphi}_{t}\right\|_{\mathcal{B}_{l_{0}}} \leq l_{0} r+\|\varphi\|_{\mathcal{B}_{l_{0}}}=r^{\prime}
$$

Define $\tilde{\Psi}: \mathcal{B}_{l_{a}}^{0} \rightarrow \mathcal{B}_{l_{a}}^{0}$ by

$$
(\tilde{\Psi} z)(t)=\left\{\begin{array}{rrr}
0, & t \in(-\infty, 0], \\
\sum_{i=1}^{n} c_{i} \varpi(t) K g\left(t_{i}, z_{t_{i}}+\tilde{\varphi}_{t_{i}}\right)+g\left(t, z_{t}+\tilde{\varphi}_{t}\right) & \\
& +\sum_{i=1}^{n} c_{i} \varpi(t) K \int_{0}^{t_{i}}\left(t_{i}-s\right)^{q-1} v\left(t_{i}-s\right) f\left(s, z_{s}+\tilde{\varphi}_{s}, \Re x(s)\right) d s & \\
& +\int_{0}^{t}(t-s)^{q-1} v(t-s) f\left(s, z_{s}+\tilde{\varphi}_{s}, \Re x(s)\right) d s & \\
& +\sum_{i=1}^{n} c_{i} \varpi(t) K \int_{0}^{t_{i}}\left(t_{i}-s\right)^{q-1} v\left(t_{i}-s\right) B u(s) d s & \\
& +\int_{0}^{t}(t-s)^{q-1} v(t-s) B u(s) d s, & t \in J .
\end{array}\right.
$$

Clearly, the operator $\Psi$ to have a fixed point is equivalent to $\tilde{\Psi}$ having one.

In view of Lemmas 2.2, 2.5 and Definition 3.2, we obtain the following lemmas, which will be useful in the proofs of the main results.

Lemma 3.3 Under the hypotheses (H2)(1), (H3)(3) and (H4)(1), for any $z \in B_{r}$, we have

$$
\|\mathcal{P}(t ; z)\| \leq N_{1} N_{2}\left\|x_{1}\right\|+N_{u}\left[d_{3}\left\|S_{r}\right\|_{L^{\frac{1}{q_{3}}}}+\frac{\Gamma(q)}{N} H_{1}\left(1+r^{\prime}\right)\right]+S_{r}(t), \quad t \in J,
$$




$$
\begin{aligned}
\|\tilde{\mathcal{P}}(z)\| \leq & \frac{a^{q}}{q} \sum_{i=1}^{n}\left|c_{i}\right| N_{u}\left[\left\|x_{1}\right\|+\frac{H_{1}\left(1+r^{\prime}\right)}{1-N \sum_{i=1}^{n}\left|c_{i}\right|}\right]+\frac{N d_{3} \sum_{i=1}^{n}\left|c_{i}\right|}{\Gamma(q)\left(1-N \sum_{i=1}^{n}\left|c_{i}\right|\right)} \\
& \cdot\left(\frac{N_{u} a^{q}}{q}+1\right)\left\|S_{r}\right\|_{L^{\frac{1}{q_{3}}}} .
\end{aligned}
$$

Here $N_{u}=\frac{N N_{1} N_{2}}{\Gamma(q)\left(1-N \sum_{i=1}^{n}\left|c_{i}\right|\right)}$.

Proof By Lemmas 2.2, 2.5 and Definition 3.2, for any $t \in J$ and $z \in B_{r}$, it is easy to get

$$
\begin{aligned}
\|B u(t ; z)\| \leq & N_{1} N_{2}\left\{\left\|x_{1}\right\|+\left\|\varpi(a) \sum_{i=1}^{n} c_{i} K g\left(t_{i}, z_{t_{i}}+\tilde{\varphi}_{t_{i}}\right)\right\|+\left\|g\left(a, z_{a}+\tilde{\varphi}_{a}\right)\right\|\right. \\
& +\left\|\varpi(a) \sum_{i=1}^{n} c_{i} K \int_{0}^{t_{i}}\left(t_{i}-s\right)^{q-1} v\left(t_{i}-s\right) f\left(s, z_{s}+\tilde{\varphi}_{s}, \Re x(s)\right) d s\right\| \\
& \left.+\left\|\int_{0}^{a}(a-s)^{q-1} v(a-s) f\left(s, z_{s}+\tilde{\varphi}_{s}, \Re x(s)\right) d s\right\|\right\} \\
\leq & N_{1} N_{2}\left\|x_{1}\right\|+\frac{N N_{1} N_{2} \sum_{i=1}^{n}\left|c_{i}\right|}{1-N \sum_{i=1}^{n}\left|c_{i}\right|} \frac{N}{\Gamma(q)} \int_{0}^{t_{i}}\left(t_{i}-s\right)^{q-1} S_{r}(s) d s \\
& +\frac{N N_{1} N_{2}}{\Gamma(q)} \int_{0}^{a}(a-s)^{q-1} S_{r}(s) d s \\
& +\frac{N N_{1} N_{2} \sum_{i=1}^{n}\left|c_{i}\right|}{1-N \sum_{i=1}^{n}\left|c_{i}\right|} H_{1}\left(1+r^{\prime}\right)+N_{1} N_{2} H_{1}\left(1+r^{\prime}\right) \\
\leq & N_{1} N_{2}\left\|x_{1}\right\|+N_{u}\left[d_{3}\left\|S_{r}\right\|_{L^{\frac{1}{q_{3}}}}+\frac{\Gamma(q) H_{1}\left(1+r^{\prime}\right)}{N}\right]
\end{aligned}
$$

and

$$
\|\mathcal{P}(t ; z)\| \leq N_{1} N_{2}\left\|x_{1}\right\|+N_{u}\left[d_{3}\left\|S_{r}\right\|_{L^{\frac{1}{q_{3}}}}+\frac{\Gamma(q) H_{1}\left(1+r^{\prime}\right)}{N}\right]+S_{r}(t)
$$

Further, we obtain

$$
\begin{aligned}
\|\tilde{\mathcal{P}}(z)\| \leq & \frac{N \sum_{i=1}^{n}\left|c_{i}\right|}{\Gamma(q)\left(1-N \sum_{i=1}^{n}\left|c_{i}\right|\right)} \int_{0}^{t_{i}}\left(t_{i}-s\right)^{q-1}\|\mathcal{P}(s ; z)\| d s \\
\leq & \frac{N \sum_{i=1}^{n}\left|c_{i}\right|}{\Gamma(q)\left(1-N \sum_{i=1}^{n}\left|c_{i}\right|\right)} \int_{0}^{t_{i}}\left(t_{i}-s\right)^{q-1}\left\{N_{1} N_{2}\left\|x_{1}\right\|\right. \\
& \left.+N_{u}\left[d_{3}\left\|S_{r}\right\|_{L^{\frac{1}{q_{3}}}}+\frac{\Gamma(q) H_{1}\left(1+r^{\prime}\right)}{N}\right]+S_{r}(s)\right\} d s \\
\leq & \frac{a^{q}}{q} \sum_{i=1}^{n}\left|c_{i}\right| N_{u}\left[\left\|x_{1}\right\|+\frac{H_{1}\left(1+r^{\prime}\right)}{1-N \sum_{i=1}^{n}\left|c_{i}\right|}\right] \\
& +\frac{N d_{3} \sum_{i=1}^{n}\left|c_{i}\right|}{\Gamma(q)\left(1-N \sum_{i=1}^{n}\left|c_{i}\right|\right)}\left(\frac{N_{u} a^{q}}{q}+1\right)\left\|S_{r}\right\|_{L^{\frac{1}{q_{3}}}} .
\end{aligned}
$$

This completes the proof. 
For the operator $\tilde{\Psi}$, we can obtain the following conclusion using Lemma 3.3.

Lemma 3.4 Let hypotheses (H2)(1), (H3)(1), (3) and (H4)(1) hold. Then the operator $\tilde{\Psi}$ : $B_{l_{a}}^{0} \rightarrow B_{l_{a}}^{0}$ is continuous provided that

$$
\frac{N \gamma d_{3}+\Gamma(q) H_{1} l_{0}}{\Gamma(q)\left(1-N \sum_{i=1}^{n}\left|c_{i}\right|\right)}\left(\frac{N_{u} a^{q}}{q}+1\right)<1
$$

Proof Firstly we show that $\tilde{\Psi}\left(B_{r}\right) \subset B_{r}$ for some $r>0$. If this was not true, there would exist $z \in B_{r}$ and $t_{r} \in J$ such that $\left\|\tilde{\Psi}(z)\left(t_{r}\right)\right\|>r$. From Lemmas 2.2 and 3.3, we have

$$
\begin{aligned}
r< & \left\|(\tilde{\Psi} z)\left(t_{r}\right)\right\| \\
\leq & \left\|\varpi\left(t_{r}\right) \tilde{\mathcal{P}}(z)\right\|+\left\|\int_{0}^{t_{r}}\left(t_{r}-s\right)^{q-1} v\left(t_{r}-s\right) \mathcal{P}(s ; z) d s\right\| \\
& +\left\|\varpi\left(t_{r}\right) \sum_{i=1}^{n} c_{i} K g\left(t_{i}, z_{t_{i}}+\tilde{\varphi}_{t_{i}}\right)\right\|+\left\|g\left(t_{r}, z_{t_{r}}+\tilde{\varphi}_{t_{r}}\right)\right\| \\
\leq & \left\|\varpi\left(t_{r}\right) \tilde{\mathcal{P}}(z)\right\|+\frac{N}{\Gamma(q)} \int_{0}^{t_{r}}\left(t_{r}-s\right)^{q-1}\left\{N_{1} N_{2}\left\|x_{1}\right\|\right. \\
& \left.+N_{u}\left[d_{3}\left\|S_{r}\right\|_{L^{\frac{1}{q_{3}}}}+\frac{\Gamma(q) H_{1}\left(1+r^{\prime}\right)}{N}\right]+S_{r}(s)\right\} d s+\frac{H_{1}\left(1+r^{\prime}\right)}{1-N \sum_{i=1}^{n}\left|c_{i}\right|} \\
\leq & \left\|\varpi\left(t_{r}\right) \tilde{\mathcal{P}}(z)\right\|+\frac{N N_{1} N_{2} a^{q}}{\Gamma(q+1)}\left\|x_{1}\right\|+\frac{N d_{3}}{\Gamma(q)}\left(\frac{N_{u} a^{q}}{q}+1\right)\left\|S_{r}\right\|_{L^{\frac{1}{q_{3}}}}+H_{1}\left(1+r^{\prime}\right) \frac{N_{u} a^{q}}{q} \\
& +\frac{H_{1}\left(1+r^{\prime}\right)}{1-N \sum_{i=1}^{n}\left|c_{i}\right|} \\
\leq & \frac{N N_{1} N_{2} a^{q}\left\|x_{1}\right\|}{\Gamma(q+1)\left(1-N \sum_{i=1}^{n}\left|c_{i}\right|\right)}+\frac{N d_{3}}{\Gamma(q)\left(1-N \sum_{i=1}^{n}\left|c_{i}\right|\right)}\left(\frac{N_{u} a^{q}}{q}+1\right)\left\|S_{r}\right\|_{L^{\frac{1}{q_{3}}}} \\
& +\frac{H_{1}\left(1+r^{\prime}\right)}{1-N \sum_{i=1}^{n}\left|c_{i}\right|}\left(\frac{N_{u} a^{q}}{q}+1\right) .
\end{aligned}
$$

Dividing both sides by $r$ and taking the lower limit as $r \rightarrow+\infty$, we have

$$
1 \leq \frac{N \gamma d_{3}+\Gamma(q) H_{1} l_{0}}{\Gamma(q)\left(1-N \sum_{i=1}^{n}\left|c_{i}\right|\right)}\left(\frac{N_{u} a^{q}}{q}+1\right)
$$

which is contrary to inequality (14). And thus $\tilde{\Psi}\left(B_{r}\right) \subset B_{r}$ for some $r>0$.

Next, we show that $\tilde{\Psi}: B_{r} \rightarrow B_{r}$ is continuous. So we take $\left\{z^{(n)}\right\}_{n \in N} \subset B_{r}$ and $z^{(n)} \rightarrow z \in$ $B_{r}$ as $n \rightarrow \infty$. Let $\mathcal{F}_{n}(s)=f\left(s, z_{s}^{(n)}+\tilde{\varphi}_{s}, \Re z^{(n)}(s)\right)$ and $\mathcal{F}(s)=f\left(s, z_{s}+\tilde{\varphi}_{s}, \Re z(s)\right)$. By (H2)(1), (H3)(1), (3), (H4)(1) and the Lebesgue dominated convergence theorem, for any $t \in J$, we get

$$
\int_{0}^{t}(t-s)^{q-1}\left\|\mathcal{F}_{(n)}(s)-\mathcal{F}(s)\right\| d s \rightarrow 0 \quad(n \rightarrow+\infty)
$$


and

$$
\begin{aligned}
\left\|u\left(t ; z^{(n)}\right)-u(t ; z)\right\| \leq & \frac{N^{2} N_{2} \sum_{i=1}^{n}\left|c_{i}\right|}{\Gamma(q)\left(1-N \sum_{i=1}^{n}\left|c_{i}\right|\right)} \int_{0}^{t_{i}}\left(t_{i}-s\right)^{q-1}\left\|\mathcal{F}_{n}(s)-\mathcal{F}(s)\right\| d s \\
& +\frac{N N_{2}}{\Gamma(q)} \int_{0}^{a}(a-s)^{q-1}\left\|\mathcal{F}_{n}(s)-\mathcal{F}(s)\right\| d s+N_{2} H_{2}\left\|z^{(n)}-z\right\| \\
& +\frac{N N_{2} \sum_{i=1}^{n}\left|c_{i}\right|}{1-N \sum_{i=1}^{n}\left|c_{i}\right|} H_{2}\left\|z^{(n)}-z\right\| \rightarrow 0 \quad(n \rightarrow+\infty) .
\end{aligned}
$$

Therefore,

$$
\begin{aligned}
&\left\|\mathcal{P}\left(t ; z^{(n)}\right)-\mathcal{P}(t ; z)\right\| \leq N_{1}\left\|u\left(t ; z^{(n)}\right)-u(t ; z)\right\|+\left\|\mathcal{F}_{n}(t)-\mathcal{F}(t)\right\| \\
& \rightarrow 0 \quad(n \rightarrow+\infty), \\
&\left\|\tilde{\mathcal{P}}\left(z^{(n)}\right)-\tilde{\mathcal{P}}(z)\right\| \leq \frac{N \sum_{i=1}^{n}\left|c_{i}\right|}{\Gamma(q)\left(1-N \sum_{i=1}^{n}\left|c_{i}\right|\right)} \int_{0}^{t_{i}}\left(t_{i}-s\right)^{q-1}\left\|\mathcal{P}\left(s ; z^{(n)}\right)-\mathcal{P}(s ; z)\right\| d s \\
& \rightarrow 0 \quad(n \rightarrow+\infty) .
\end{aligned}
$$

Finally, we have

$$
\begin{aligned}
\left\|\left(\tilde{\Psi} z^{(n)}\right)(t)-(\tilde{\Psi} z)(t)\right\| \leq & N\left\|\tilde{\mathcal{P}}\left(z^{(n)}\right)-\tilde{\mathcal{P}}(z)\right\| \\
& +\frac{N}{\Gamma(q)} \int_{0}^{t}(t-s)^{q-1}\left\|\mathcal{P}\left(s ; z^{(n)}\right)-\mathcal{P}(s ; z)\right\| d s \\
& +\frac{N \sum_{i=1}^{n}\left|c_{i}\right|}{1-N \sum_{i=1}^{n}\left|c_{i}\right|}\left\|g\left(t_{i} ; z_{t_{i}}^{(n)}\right)-g\left(t_{i} ; z_{t_{i}}\right)\right\| \\
& +\left\|g\left(t ; z_{t}^{(n)}\right)-g\left(t ; z_{t}\right)\right\| \\
\leq & N\left\|\tilde{\mathcal{P}}\left(z^{(n)}\right)-\tilde{\mathcal{P}}(z)\right\| \\
& +\frac{N}{\Gamma(q)} \int_{0}^{t}(t-s)^{q-1}\left\|\mathcal{P}\left(s ; z^{(n)}\right)-\mathcal{P}(s ; z)\right\| d s \\
& +\frac{H_{2} N \sum_{i=1}^{n}\left|c_{i}\right|}{1-N \sum_{i=1}^{n}\left|c_{i}\right|}\left\|z^{(n)}-z\right\| \\
& +H_{2}\left\|z^{(n)}-z\right\| \rightarrow 0 \quad(n \rightarrow+\infty) .
\end{aligned}
$$

Hence the given operator $\tilde{\Psi}: B_{r} \rightarrow B_{r}$ is continuous. And the proof is completed.

Now, we present and prove the controllability conclusions for the fractional neutral control system (1) with infinite delay and nonlocal conditions.

Theorem 3.1 If the hypotheses $(\mathrm{H} 0)-(\mathrm{H} 4)$ are satisfied, then the fractional neutral nonlocal system (1) with the initial problem $x(0)=\sum_{i=1}^{n} c_{i} x\left(t_{i}\right)+g\left(0, x_{0}\right), x_{0}=\varphi \in \mathcal{B}_{l_{0}}$ is controllable on J provided that (14) and

$$
\alpha=\frac{2 N N_{4}\left(1+2 \Upsilon^{*}\right) \sum_{i=1}^{n}\left|c_{i}\right|+\Gamma(q) \kappa}{\Gamma(q)\left(1-N \sum_{i=1}^{n}\left|c_{i}\right|\right)}\left(N_{3} N_{5}+1\right)<1,
$$

where $N_{3}=d_{1}\left\|\xi_{1}\right\|_{L^{\frac{1}{q_{1}}}} ; N_{4}=d_{2}\left\|\xi_{2}\right\|_{L^{\frac{1}{q_{2}}}} ; N_{5}=\frac{2 N N_{1}}{\Gamma(q)\left(1-N \sum_{i=1}^{n}\left|c_{i}\right|\right)}$. 
Proof We have proved $\tilde{\Psi}: B_{r} \rightarrow B_{r}$ is continuous in Lemma 3.4. Furthermore, we prove that $\tilde{\Psi}\left(B_{r}\right)$ is equicontinuous on $J$. Indeed, let $z \in \tilde{\Psi}\left(B_{r}\right)$ and $0 \leq t^{\prime}<t^{\prime \prime} \leq a$. and take the following notations:

$$
\begin{aligned}
& I_{1}=\left\|\varpi\left(t^{\prime \prime}\right) \tilde{\mathcal{P}}(z)-\varpi\left(t^{\prime}\right) \tilde{\mathcal{P}}(z)\right\|, \\
& I_{2}=\left\|\int_{0}^{t^{\prime}}\left[\left(t^{\prime \prime}-s\right)^{q-1}-\left(t^{\prime}-s\right)^{q-1}\right] v\left(t^{\prime \prime}-s\right) \mathcal{P}(s ; z) d s\right\|, \\
& I_{3}=\left\|\int_{0}^{t^{\prime}}\left(t^{\prime}-s\right)^{q-1}\left[v\left(t^{\prime \prime}-s\right)-v\left(t^{\prime}-s\right)\right] \mathcal{P}(s ; z) d s\right\|, \\
& I_{4}=\left\|\int_{t^{\prime}}^{t^{\prime \prime}}\left(t^{\prime \prime}-s\right)^{q-1} v\left(t^{\prime \prime}-s\right) \mathcal{P}(s ; z) d s\right\|, \\
& I_{5}=\left\|\varpi\left(t^{\prime \prime}\right) \sum_{i=1}^{n} c_{i} K g\left(t_{i}, z_{t_{i}}+\tilde{\varphi}_{t_{i}}\right)-\varpi\left(t^{\prime}\right) \sum_{i=1}^{n} c_{i} K g\left(t_{i}, z_{t_{i}}+\tilde{\varphi}_{t_{i}}\right)\right\|, \\
& I_{6}=\left\|g\left(t^{\prime \prime}, z_{t^{\prime \prime}}+\tilde{\varphi}_{t^{\prime \prime}}\right)-g\left(t^{\prime}, z_{t^{\prime}}+\tilde{\varphi}_{t^{\prime}}\right)\right\| .
\end{aligned}
$$

So we can write

$$
\left\|\left(\tilde{\Psi}(z)\left(t^{\prime \prime}\right)\right)-\left(\tilde{\Psi}(z)\left(t^{\prime}\right)\right)\right\| \leq I_{1}+I_{2}+I_{3}+I_{4}+I_{5}+I_{6}
$$

Obviously, we have

$$
I_{5} \leq \frac{H_{1}\left(1+r^{\prime}\right) \sum_{i=1}^{n}\left|c_{i}\right|}{1-N \sum_{i=1}^{n}\left|c_{i}\right|}\left\|\varpi\left(t^{\prime \prime}\right)-\varpi\left(t^{\prime}\right)\right\|
$$

The hypothesis (H1) can ensure $I_{1} \rightarrow 0$ and $I_{5} \rightarrow 0$ as $t^{\prime \prime}-t^{\prime} \rightarrow 0$. Using Lemmas 2.2 and 3.3, we can obtain

$$
\begin{aligned}
I_{2} \leq & {\left[\frac{N}{\Gamma(q)}\left(N_{1} N_{2}\left\|x_{1}\right\|+N_{u} d_{3}\left\|S_{r}\right\|_{L^{\frac{1}{q_{3}}}}\right)+N_{u} H_{1}\left(1+r^{\prime}\right)\right] \int_{0}^{t^{\prime}}\left|\left(t^{\prime \prime}-s\right)^{q-1}-\left(t^{\prime}-s\right)^{q-1}\right| d s } \\
& +\frac{N\left\|S_{r}\right\|_{L^{\frac{1}{q_{3}}}}}{\Gamma(q)}\left(\int_{0}^{t^{\prime}}\left|\left(t^{\prime \prime}-s\right)^{q-1}-\left(t^{\prime}-s\right)^{q-1}\right| \frac{1}{1-q_{3}} d s\right)^{1-q_{3}}, \\
I_{4} \leq & {\left[\frac{N}{\Gamma(q+1)}\left(N_{1} N_{2}\left\|x_{1}\right\|+N_{u} d_{3}\left\|S_{r}\right\|_{L^{\frac{1}{q_{3}}}}\right)+\frac{N_{u} H_{1}\left(1+r^{\prime}\right)}{q}\right]\left(t^{\prime \prime}-t^{\prime}\right)^{q} } \\
& +\frac{N\left\|S_{r}\right\|_{L^{\frac{1}{q_{3}}}}}{\Gamma(q)\left(b_{3}+1\right)^{1-q_{3}}}\left(t^{\prime \prime}-t^{\prime}\right)^{q-q_{3}},
\end{aligned}
$$

which indicates that $I_{2} \rightarrow 0$ and $I_{4} \rightarrow 0$ as $t^{\prime \prime}-t^{\prime} \rightarrow 0$. If $t^{\prime} \equiv 0,0<t^{\prime \prime} \leq a$, it is obvious that $I_{3} \equiv 0$. For $t^{\prime}>0$ and $\delta\left(0<\delta<t^{\prime}\right)$ small enough, we obtain

$$
\begin{aligned}
I_{3} \leq & \left\|\int_{0}^{t^{\prime}-\delta}\left(t^{\prime}-s\right)^{q-1}\left[v\left(t^{\prime \prime}-s\right)-v\left(t^{\prime}-s\right)\right] \mathcal{P}(s ; z) d s\right\| \\
& +\left\|\int_{t^{\prime}-\delta}^{t^{\prime}}\left(t^{\prime}-s\right)^{q-1}\left[v\left(t^{\prime \prime}-s\right)-v\left(t^{\prime}-s\right)\right] \mathcal{P}(s ; z) d s\right\|
\end{aligned}
$$




$$
\begin{aligned}
\leq & \left\{\frac{\left[N_{1} N_{2}\left\|x_{1}\right\|+N_{u} d_{3}\left\|S_{r}\right\|_{L^{\frac{1}{q_{3}}}}+\frac{\Gamma(q)}{N} N_{u} H_{1}\left(1+r^{\prime}\right)\right]\left(\left(t^{\prime}\right)^{q}-\delta^{q}\right)}{q}\right. \\
& \left.+\frac{\left\|S_{r}\right\|_{L^{\frac{1}{q_{3}}}}\left(\left(t^{\prime}\right)^{q-q_{3}}-\delta^{q-q_{3}}\right)}{\left(b_{3}+1\right)^{1-q_{3}}}\right\} \\
& \cdot \sup _{s \in\left[0, t^{\prime}-\delta\right]}\left\|v\left(t^{\prime \prime}-s\right)-v\left(t^{\prime}-s\right)\right\|+\frac{2 N\left\|S_{r}\right\|_{L^{\frac{1}{q_{3}}}} \delta^{q-q_{3}}}{\Gamma(q)\left(b_{3}+1\right)^{1-q_{3}}} \\
& +\frac{2 N\left[N_{1} N_{2}\left\|x_{1}\right\|+N_{u} d_{3}\left\|S_{r}\right\|_{L^{\frac{1}{q_{3}}}}+\frac{\Gamma(q)}{N} N_{u} H_{1}\left(1+r^{\prime}\right)\right] \delta^{q}}{\Gamma(q+1)} .
\end{aligned}
$$

It follows from the assumption (H1) that $I_{3} \rightarrow 0$ as $t^{\prime \prime}-t^{\prime} \rightarrow 0$ and $\delta \rightarrow 0$. From (H4)(1), we obtain

$$
I_{6} \leq H_{2}\left(\left\|z_{t^{\prime \prime}}-z_{t^{\prime}}\right\|+\left\|\varphi_{t^{\prime \prime}}-\varphi_{t^{\prime}}\right\|\right)+H_{3}\left|t^{\prime \prime}-t^{\prime}\right|
$$

Since $z \in B_{r}$, we get $I_{6} \rightarrow 0$ as $t^{\prime \prime}-t^{\prime} \rightarrow 0$. Thus $\tilde{\Psi}\left(B_{r}\right)$ is equicontinuous on $(-\infty, a]$.

Next we will verify that $\tilde{\Psi}$ satisfies Mönch's condition. Assume that $D \subset B_{r}$ is countable and $D \subset \overline{\operatorname{co}}(\{0\} \cup \tilde{\Psi}(D))$, we show that $\chi(D)=0$.

It follows from (H2)(2), (H3)(2) and (H4)(2) that

$$
\begin{aligned}
& \chi(B u(s ; W)) \leq\left[N_{4} N_{5}\left(1+2 \Upsilon^{*}\right)+\frac{N_{1} \kappa}{1-N \sum_{i=1}^{n}\left|c_{i}\right|}\right] \xi_{1}(s) \chi(W) \\
& \chi(\mathcal{P}(s ; W)) \leq\left[N_{4} N_{5}\left(1+2 \Upsilon^{*}\right)+\frac{N_{1} \kappa}{1-N \sum_{i=1}^{n}\left|c_{i}\right|}\right] \xi_{1}(s) \chi(W)+\left(1+2 \Upsilon^{*}\right) \xi_{2}(s) \chi(W),
\end{aligned}
$$

and

$$
\chi(\tilde{\mathcal{P}}(W)) \leq\left[\frac{2 N N_{4}\left(1+2 \Upsilon^{*}\right) \sum_{i=1}^{n}\left|c_{i}\right|}{\Gamma(q)\left(1-N \sum_{i=1}^{n}\left|c_{i}\right|\right)}\left(N_{3} N_{5}+1\right)+\frac{N_{3} N_{5} \sum_{i=1}^{n}\left|c_{i}\right| \kappa}{1-N \sum_{i=1}^{n}\left|c_{i}\right|}\right] \chi(W),
$$

for $s \in[0, t], t \in J$. Furthermore, we obtain

$$
\begin{aligned}
\chi(\tilde{\Psi}(W)(t)) & \leq N \chi(\tilde{\mathcal{P}}(W))+\frac{2 N}{\Gamma(q)} \int_{0}^{t}(t-s)^{q-1} \chi(\mathcal{P}(s ; W)) d s+\frac{\kappa}{1-N \sum_{i=1}^{n}\left|c_{i}\right|} \chi(w) \\
& \leq \frac{2 N N_{4}\left(1+2 \Upsilon^{*}\right) \sum_{i=1}^{n}\left|c_{i}\right|+\Gamma(q) \kappa}{\Gamma(q)\left(1-N \sum_{i=1}^{n}\left|c_{i}\right|\right)}\left(N_{3} N_{5}+1\right) \chi(W)=\alpha \chi(W) .
\end{aligned}
$$

Combining the equicontinuity and boundedness of $\tilde{\Psi}(W)$, we obtain

$$
\chi(\tilde{\Psi}(W))=\max _{t \in J} \chi(\tilde{\Psi}(W)(t)) \leq \alpha \chi(W) .
$$

Hence,

$$
\chi(W) \leq \chi(\overline{\operatorname{co}}(\{0\} \cup \tilde{\Psi}(W))) \leq \chi(\tilde{\Psi}(W)) \leq \alpha \chi(W) .
$$

From the inequality (15) $\alpha<1$, we have $\chi(W)=0$. That is, $W$ is relatively compact. Therefore using Lemma 2.6, $\tilde{\Psi}$ has at least one fixed point $z$ in $B_{r}$. Then $x=z+\tilde{\varphi}$ is a mild solution 
of the system (1) and satisfies $x(a)=x_{1}$. Thus, the fractional neutral nonlocal system (1) is controllable on $J$. The proof is completed.

Corollary 3.1 The hypothesis $(\mathrm{H} 3)(3)$ can be replaced by

(H3) (3)' For each $r>0$, there exist a constant $q_{3} \in(0, q)$ and $\bar{S} \in L^{\frac{1}{q_{3}}}\left(J, R^{+}\right)$such that

$$
\sup \left\{\|f(t, \varphi, x)\|:\|\varphi\|_{B_{l_{0}}} \leq r^{\prime},\|y\| \leq \Upsilon^{*} r\right\} \leq \bar{S}(t), \quad t \in J
$$

where $\varphi \in B_{l_{0}}$ s.t. $x(0)=\sum_{i=1}^{n} c_{i} x\left(t_{i}\right)+g\left(0, x_{0}\right)$. (H3)(1), (2) are not changed. Thus assume the hypotheses (H0)-(H3)(1), (2), (3)' and (H4) hold, (14) and (15) are established, the system (1) is also controllable on $J$.

Proof The proof is similar to Theorem 3.1.

\section{Applications}

Example 4.1 Consider the following fractional neutral evolution equations:

$$
\left\{\begin{array}{l}
\frac{\frac{\partial}{4}^{\frac{3}{4}}}{\partial t^{\frac{3}{4}}}\left[x(t, v)-t \int_{-\infty}^{0} \zeta(\tau) \frac{|x(t+\tau, v)|}{1+|x(t+\tau, v)|} d \tau\right] \\
\quad=\frac{\partial}{\partial t}\left[x(t, v)-t \int_{-\infty}^{0} \zeta(\tau) \frac{|x(t+\tau, v)|}{1+|x(t+\tau, v)|} d \tau\right] \\
\quad \quad \quad \frac{e^{-2 t}}{1+e^{t}}\left[x(t+\tau, v)+\int_{0}^{t}(t-s)^{2} x(s, v) d s\right]+\lambda \rho(t, v), \quad 0 \leq t \leq a, 0 \leq v \leq 1, \\
x(t, 0)-t \int_{-\infty}^{0} \zeta(\tau) \frac{|x(t+\tau, 0)|}{1+|x(t+\tau, 0)|} d \tau=0, \quad 0 \leq t \leq a, \\
x(t, 1)-t \int_{-\infty}^{0} \zeta(\tau) \frac{|x(t+\tau, 1)|}{1+|x(t+\tau, 1)|} d \tau=0, \quad 0 \leq t \leq a, \\
x(0, v)=\sum_{i=1}^{n} \arctan \frac{1}{2 i^{2}} x(i, v), \quad 0 \leq v \leq 1,
\end{array}\right.
$$

where $\lambda>0$ and $0<n<a . \rho:[0, a] \times(0,1) \rightarrow(0,1), \zeta:(-\infty, 0] \rightarrow R$ and $x_{0}:(-\infty, 0] \times$ $(0,1) \rightarrow R$ are continuous functions, and $\int_{-\infty}^{0}|\zeta(\tau)| d \tau<\infty$.

Let $E=U=: C([0,1])$ and $A$ be defined by

$$
\left\{\begin{array}{l}
D(A)=\left\{w \in E: w^{\prime} \in E, w(0)=w(1)=0\right\} \\
A w=-w^{\prime}, \quad w \in D(A)
\end{array}\right.
$$

As is well known, $-A$ generates an equicontinuous semigroup $T(t)(t \geq 0)$ in $E$ and it satisfies

$$
T(t) w(s)=w(t+s)
$$

for $w \in E$. Thus $T(t)(t \geq 0)$ is not compact in $E$ and $\sup _{0 \leq t \leq a}\|T(t)\| \leq 1$. Take

$$
\begin{aligned}
& x(t)(v)=x(t, v), \\
& D^{\frac{3}{4}} x(t)(v)=\frac{\partial^{\frac{3}{4}}}{\partial t^{\frac{3}{4}}} x(t, v), \\
& g\left(t, x_{t}\right)(v)=t \int_{-\infty}^{0} \zeta(\tau) \frac{\left|x_{t}(\tau)(v)\right|}{1+\left|x_{t}(\tau)(v)\right|} d \tau, \\
& f\left(t, x_{t}, \Re x(t)\right)(v)=\frac{e^{-2 t}}{1+e^{t}}\left[x(t+\tau, v)+\int_{0}^{t}(t-s)^{2} x(s, v) d s\right],
\end{aligned}
$$




$$
\begin{aligned}
& u(t) v=\rho(t, v), \\
& c_{i}=\arctan \frac{1}{2 i^{2}}, \quad t_{i}=i, \quad i=1,2, \ldots, n .
\end{aligned}
$$

Define the norm by

$$
\|y\|_{B_{l_{0}}}=\int_{-\infty}^{0} l(s)\|\varphi\|_{[s, 0]} d s, \quad y \in B_{l_{0}} .
$$

Then, for any $x \in B_{r}, t \in J$, we obtain

$$
\begin{aligned}
\left\|f\left(t, x_{t}, \Re x(t)\right)(z)\right\| & \leq \frac{e^{-2 t}}{1+e^{t}}\left[\|x(t+\tau, z)\|+\int_{0}^{t}\left\|(t-s)^{2} x(s, z)\right\| d s\right] \\
& \leq \frac{\left(3+a^{3}\right) e^{-2 t} r^{\prime}}{3\left(1+e^{t}\right)} \\
& \leq \frac{\left(3+a^{3}\right) r^{\prime}}{6} .
\end{aligned}
$$

Thus, the hypothesis (H3) holds for $\beta=\frac{3+a^{3}}{6}$ and $\xi_{2}(t)=\frac{1}{2}$ for all $t \in J$. By

$$
\sum_{i=1}^{n}\left|c_{i}\right| \leq \sum_{i=1}^{\infty} \arctan \frac{1}{2 i^{2}}=\frac{\pi}{4}<1
$$

we verify that the hypothesis (H0) holds.

For $t, t^{\prime}, t^{\prime \prime} \in[0, a], z, y \in B_{r}$, we have

$$
\begin{aligned}
& \|g(t, z)\|=\left\|t \int_{-\infty}^{0} \zeta(\tau) \frac{|z(\tau)(v)|}{1+|z(\tau)(v)|} d \tau\right\| \leq a \int_{-\infty}^{0}|\zeta(\tau)|\|1+|z(\tau)(v)|\| d \tau \\
& \leq H_{1}\left(1+r^{\prime}\right) \text {, } \\
& \left\|g\left(t^{\prime}, z\right)-g\left(t^{\prime \prime}, y\right)\right\| \leq\left|t^{\prime}-t^{\prime \prime}\right| \int_{-\infty}^{0}\left\|\zeta(\tau) \frac{|z(\tau)(v)|}{1+|z(\tau)(v)|}\right\| d \tau \\
& +t^{\prime \prime} \int_{-\infty}^{0}\left\|\zeta(\tau)\left[\frac{|z(\tau)(v)|}{1+|z(\tau)(v)|}-\frac{|y(\tau)(v)|}{1+|y(\tau)(v)|}\right]\right\| d \tau \\
& \leq\left|t^{\prime}-t^{\prime \prime}\right| \int_{-\infty}^{0}|\zeta(\tau)| d \tau+a \int_{-\infty}^{0}|\zeta(\tau)| d \tau\|z-y\| \\
& =H_{2}\|z-y\|+H_{3}\left|t^{\prime}-t^{\prime \prime}\right| \text {, } \\
& \|g(t, z)-g(t, y)\| \leq t \int_{-\infty}^{0}\left\|\zeta(\tau)\left[\frac{|z(\tau)(v)|}{1+|z(\tau)(v)|}-\frac{|y(\tau)(v)|}{1+|y(\tau)(v)|}\right]\right\| d \tau \\
& \leq t \int_{-\infty}^{0}|\zeta(\tau)| d \tau\|z-y\|
\end{aligned}
$$

where $H_{1}=H_{2}=a \int_{-\infty}^{0}|\zeta(\tau)| d \tau, H_{3}=\int_{-\infty}^{0}|\zeta(\tau)| d \tau$. Therefore, for any countable set $W \subset$ $B_{r}$, we obtain

$$
\chi(g(t, W)) \leq t \int_{-\infty}^{0}|\zeta(\tau)| d \tau \sup _{-\infty<\tau \leq 0} \chi(W(\tau))
$$

where $\xi_{3}(t)=t \int_{-\infty}^{0}|\zeta(\tau)| d \tau$ 
For $v \in(0,1)$, the operator $\Im$ is defined by

$$
\begin{aligned}
(\Im(t))(v)= & \varpi(a)\left[I-\sum_{i=1}^{n} \arctan \frac{1}{2 i^{2}} \varpi(i)\right]^{-1} \sum_{i=1}^{n} \arctan \frac{1}{2 i^{2}} \int_{0}^{i}(i-s)^{-\frac{1}{4}} \\
& \cdot v(i-s) \lambda \rho(s, v) d s+\int_{0}^{a}(a-s)^{-\frac{1}{4}} v(a-s) \lambda \rho(s, v) d s
\end{aligned}
$$

where $\{\varpi(t)\}_{(t \geq 0)}$ and $\{v(t)\}_{(t \geq 0)}$ satisfy

$$
\begin{aligned}
& \varpi(t) w(s)=\int_{0}^{\infty} \frac{4}{3} \vartheta^{-\frac{7}{3}} \varrho_{\frac{3}{4}}\left(-\vartheta^{-\frac{4}{3}}\right) w\left(t^{\frac{3}{4}} \vartheta+s\right) d \vartheta \\
& \nu(t) w(s)=\frac{3}{4} \int_{0}^{\infty} \frac{4}{3} \vartheta^{-\frac{4}{3}} \varrho_{\frac{3}{4}}\left(-\vartheta^{-\frac{4}{3}}\right) w\left(t^{\frac{3}{4}} \vartheta+s\right) d \vartheta
\end{aligned}
$$

and $\varrho_{\frac{3}{4}}$ is given by $\varrho_{\frac{3}{4}}=\frac{1}{\pi} \sum_{n=1}^{\infty}(-1)^{n-1} \vartheta^{-\frac{3}{4} n-1} \frac{\Gamma\left(\frac{3}{4} n+1\right)}{n !} \sin \left(\frac{3 n \pi}{4}\right), \vartheta \in(0, \infty)$. If we let $\Im$ satisfy the hypothesis $(\mathrm{H} 2)$, from Theorem 3.1, we see that the system (16) is controllable on $[0, a]$ provided that (14) and (15).

Example 4.2 To illustrate the application of the theory we consider another partial integro-differential equation, with fractional derivative of the form

$$
\left\{\begin{array}{l}
\frac{\partial^{\frac{3}{4}}}{\partial t^{\frac{3}{4}}}\left[x(t, v)-\int_{-\infty}^{t} e^{(s-t)}(x(s, v)) d s\right] \\
\quad=\frac{\partial}{\partial t}\left[x(t, v)-\int_{-\infty}^{t} e^{(s-t)}(x(s, v)) d s\right]+\int_{-\infty}^{t} H(t, v, s-t) Q(x(s, v)) d s \\
\quad+\int_{0}^{t} \Upsilon(s, t) e^{-x(s, v)} d s+\lambda \rho(t, v), \quad 0 \leq t \leq a, 0 \leq v \leq 1, \\
x(t, 0)=x(t, 1)=0, \quad 0 \leq t \leq a, \\
x(0, v)=\sum_{i=1}^{n} \arctan \frac{1}{2 i^{2}} x(i, v), \quad 0 \leq v \leq 1
\end{array}\right.
$$

where $\varphi \in B_{l_{0}}, \lambda>0,0<n<a$ and $\rho:[0, a] \times(0,1) \rightarrow(0,1)$.

Let $E=U=: L^{2}([0,1])$ and let $A: D(A) \subset E \rightarrow E$ be defined by $A w=-w^{\prime}, w \in D(A)$, where $D(A)=\left\{w \in E: w^{\prime} \in E, w(0)=w(1)=0\right\}$. It is well known that $-A$ is an infinitesimal generator of a semigroup $T(t)(t \geq 0)$ in $E$ and is given by $T(t) w(s)=w(t+s)$ for $w \in E$. Thus $T(t)(t \geq 0)$ is not compact in $E$ with $\chi(T(t) D) \leq \chi(D)$ where $\chi$ is the Hausdorff MNC and there exists a $N$ such that $\sup _{0 \leq t \leq a}\|T(t)\| \leq N$. Moreover, $t \rightarrow w\left(t^{\frac{3}{4}} \vartheta+s\right) x$ is equicontinuous for $t>0$ and $\vartheta \in(-\infty, 0)$.

Let $l(s)=e^{s}, s<0$, then $l_{0}=\int_{-\infty}^{0} l(s) d s=1$, and we define

$$
\|\varphi\|_{B_{l_{0}}}=\int_{-\infty}^{0} l(s) \sup _{\tau \in[s, 0]}\|\varphi(\tau)\| d s .
$$

Let $x:(-\infty, a] \rightarrow R$ be such that $x_{0} \in B_{l_{0}}$. For $t \in[0, a]$, we have

$$
\left\|x_{t}\right\|_{B_{l_{0}}}=\int_{-\infty}^{0} l(s) \sup _{\tau \in[s, 0]}\left\|x_{t}(\tau)\right\| d s \leq \sup _{s \in[0, t]}|x(s)|+\left\|x_{0}\right\|_{B_{l_{0}}}<\infty .
$$

Hence $x_{t} \in B_{l_{0}}$. Now we prove that

$$
\left\|x_{t}\right\|_{B_{l_{0}}} \leq K(t) \sup _{s \in[0, t]}|x(s)|+M(t)\left\|x_{0}\right\|_{B_{l_{0}}},
$$

where $K(t) \equiv M(t) \equiv 1, H=1$. 
For $\tau+t \leq 0$, we derive

$$
\left|x_{t}(\tau)\right|=|x(t+\tau)| \leq \sup \{|x(s)|:-\infty<s \leq 0\}
$$

If $\tau+t \geq 0$, then we get

$$
\left|x_{t}(\tau)\right| \leq \sup \{|x(s)|: 0 \leq s \leq 0\}
$$

Thus for all $\tau+t \in(-\infty, a]$, we obtain

$$
\left|x_{t}(\tau)\right| \leq \sup \{|x(s)|:-\infty<s \leq 0\}+\sup \{|x(s)|: 0 \leq s \leq 0\}
$$

It is clear that $\left(B_{l_{0}},\|\cdot\|_{B_{l_{0}}}\right)$ is a Banach space. We can conclude that $B_{l_{0}}$ is a phase space. Define

$$
\begin{aligned}
& x(t)(v)=x(t, v), \\
& D^{\frac{3}{4}} x(t)(v)=\frac{\partial^{\frac{3}{4}}}{\partial t^{\frac{3}{4}}} x(t, v), \\
& g(t, \varphi)(v)=\int_{-\infty}^{0} e^{\tau} \varphi(\tau)(v) d \tau \\
& f(t, \varphi, \Re x(t))(v)=\int_{-\infty}^{0} H(t, v, \tau) Q(\varphi(\tau) v) d \tau+\Re x(t)(v), \\
& c_{i}=\arctan \frac{1}{2 i^{2}}, \quad t_{i}=i, \quad i=1,2, \ldots, n,
\end{aligned}
$$

where $\Re x(t)(v)=\int_{0}^{t} \Upsilon(s, t) e^{-x(s, v)} d s$. Then with these settings equations (17) can be written in the abstract form of (1). Suppose further that:

(a) The function $H(t, x, \tau) \geq 0$ is continuous in $J \times[0,1] \times(-\infty, 0]$ and satisfies $\int_{-\infty}^{0} H^{2}(t, x, \tau) d \tau<\infty$

(b) The function $Q(\cdot)$ is continuous, $0 \leq Q(x(\tau, v)) \leq \int_{-\infty}^{0} e^{s}|x(s, \cdot)|_{L^{2}} d s$ for $(\tau, v) \in(-\infty, 0] \times[0,1]$.

Thus under the above hypotheses, we have

$$
\begin{aligned}
\|f(t, \varphi, \Re x(t))\|_{L^{2}} \leq & \left\{\int_{0}^{1}\left[\int_{-\infty}^{0} H(t, v, \tau) Q(\varphi(\tau)(v)) d \tau\right]^{2} d v\right\}^{\frac{1}{2}} \\
& +\left\{\int_{0}^{1}[B x(t)(v)]^{2} d v\right\}^{\frac{1}{2}} \\
\leq & \left\{\int_{0}^{1}\left[\int_{-\infty}^{0} H(t, v, \tau) \int_{-\infty}^{0} e^{s}|\varphi(\tau)(\cdot)|_{L^{2}} d s d \tau\right]^{2} d v\right\}^{\frac{1}{2}}+\|\Re x(t)\|_{L^{2}} \\
\leq & \left\{\int_{0}^{1}\left[\int_{-\infty}^{0} H(t, v, \tau) d \tau\right]^{2} d v\right\}^{\frac{1}{2}}\|\varphi\|_{B_{l_{0}}}+\|\Re x(t)\|_{L^{2}} \\
= & S_{r}(t),
\end{aligned}
$$

hence $f$ satisfies (H3) and in a similar way we can show that $g$ may satisfy $(\mathrm{H} 4)$. 
For $v \in(0,1)$, the operator $\Im$ is defined by

$$
\begin{aligned}
(\Im(t))(v)= & \varpi(a)\left[I-\sum_{i=1}^{n} \arctan \frac{1}{2 i^{2}} \varpi(i)\right]^{-1} \sum_{i=1}^{n} \arctan \frac{1}{2 i^{2}} \int_{0}^{i}(i-s)^{-\frac{1}{4}} \\
& \cdot v(i-s) \lambda \rho(s, v) d s+\int_{0}^{a}(a-s)^{-\frac{1}{4}} v(a-s) \lambda \rho(s, v) d s
\end{aligned}
$$

where $\{\varpi(t)\}_{(t \geq 0)}$ and $\{v(t)\}_{(t \geq 0)}$ are the same as the formulas in Example 4.1.

Then all the conditions of Theorem 3.1 are satisfied. Hence, system (17) is controllable on $J$.

\section{Competing interests}

The authors declare that they have no competing interests.

\section{Authors' contributions}

The authors have made equal contributions of this manuscript. All authors read and approved the final version.

\section{Acknowledgements}

This work is supported by National Natural Science Foundation of China (Nos. 11371027, 11471015 and 11601003), Natural Science Fund of Anhui Province (No. 1708085MA15).

\section{Publisher's Note}

Springer Nature remains neutral with regard to jurisdictional claims in published maps and institutional affiliations.

Received: 1 November 2016 Accepted: 13 April 2017 Published online: 18 May 2017

\section{References}

1. Hilfer, R: Applications of Fractional Calculus in Physics. World Scientific, Singapore (2000)

2. Diethelm, K, Freed, AD: On the solution of nonlinear fractional order differential equations used in the modeling of viscoelasticity. In: Keil, F, Mackens, W, Voss, H, Werther, J (eds.) Scientific Computing in Chemical Engineering II Computational Fluid Dynamics, Reaction Engineering and Molecular Properties. Springer, Heidelberg (1999)

3. Baleanu, D, Diethelm, K, Scalas, E, Trujillo, JJ: Fractional Calculus Models and Numerical Methods. Series on Complexity, Nonlinearity and Chaos. World Scientific, Singapore (2012)

4. Kilbas, AA, Srivastava, HM, Trujillo, JJ: Theory and Applications of Fractional Differential Equations. North-Holland Mathematics Studies. Elsevier Science BV, Amsterdam (2006)

5. Miller, KS, Ross, B: An Introduction to the Fractional Calculus and Fractional Differential Equations. Wiley, New York (1993)

6. Podlubny, I: Fractional Differential Equations. Academic Press, San Diego (1999)

7. Baleanu, D, Machado, JAT, Luo, ACJ: Fractional Dynamics and Control. Springer, London (2014)

8. El-Borai, MM: Some probability densities and fundamental solutions of fractional evolution equations. Chaos Solitons Fractals 14, 433-440 (2002)

9. Zhou, Y, Jiao, F: Existence of mild solutions for fractional neutral evolution equations. Comput. Math. Appl. 59, 1063-1077 (2010)

10. Zhou, Y, Jiao, F: Nonlocal Cauchy problem for fractional evolution equations. Nonlinear Anal., Real World Appl. 11, 4465-4475 (2010)

11. Dabas, J, Chauhan, A: Existence and uniqueness of mild solution for an impulsive neutral fractional integro-differential equation with infinite delay. Math. Comput. Model. 57, 754-763 (2013)

12. Li, F, Liang, J, Xu, H: Existence of mild solutions for fractional integro-differential equation of Sobolev type with nonlocal conditions. J. Math. Anal. Appl. 391, 510-525 (2012)

13. Chauhan, A, Dabas, J: Local and global existence of mild solution to an impulsive fractional functional integro-differential equation with nonlocal condition. Commun. Nonlinear Sci. Numer. Simul. 19(4), 821-829 (2014)

14. Ravichandran, C, Baleanu, D: On the controllability of fractional functional integro-differential systems with an infinite delay in Banach spaces. Adv. Differ. Equ. 2013, 291 (2013)

15. Balachandran, K, Park, JY: Controllability of fractional integro-differential systems in Banach spaces. Nonlinear Anal. Hybrid Syst. 3, 363-367 (2009)

16. Liang, J, Yang, H: Controllability of fractional integro-differential evolution equations with nonlocal conditions. Appl. Math. Comput. 254, 20-29 (2015)

17. Ji, S, Li, G, Wang, M: Controllability of impulsive differential systems with nonlocal conditions. Appl. Math. Comput. $217,6981-6989(2011)$

18. Wang, JR, Zhou, Y: Nonlocal controllability of semilinear dynamic systems with fractional derivative in Banach spaces. J. Optim. Theory Appl. 154(1), 292-302 (2012)

19. Mahmudov, $\mathrm{NI}$, Zorlu, S: On the approximate controllability of fractional evolution equations with compact analysis semigroup. J. Comput. Appl. Math. 259, 194-204 (2014) 
20. Liu, B: Controllability of impulsive neutral functional differential inclusions with infinite delay. Nonlinear Anal., Theory Methods Appl. 60, 1533-1552 (2005)

21. Chalishajar, DN, Malar, K, Karthikeyan, K: Approximate controllability of abstract impulsive fractional neutral evolution equations with infinite delay in Banach space. Electron. J. Differ. Equ. 2013, 275 (2013)

22. Sakthivel, R, Mahmucov, NI, Nieto, JJ: Controllability for a class of fractional-order neutral evolution control systems. Appl. Math. Comput. 218, 10334-10340 (2012)

23. Wang, JR, Zhou, Y: Complete controllability of fractional evolution systems. Commun. Nonlinear Sci. Numer. Simul. 17(11), 4346-4355 (2012)

24. Obukhovski, V, Zecca, P: Controllability for systems governed by semilinear differential inclusions in a Banach space with a noncompact semigroup. Nonlinear Anal., Theory Methods Appl. 70(9), 3424-3436 (2009)

25. Hernández, E, O'Regan, D: On the recent developments in the theory of abstract differential equations with fractional derivatives. Nonlinear Anal. 73, 3462-3472 (2010)

26. Byszewski, L: Theorems about the existence and uniqueness of solutions of a semilinear evolution nonlocal Cauchy problem. J. Math. Anal. Appl. 162, 497-505 (1991)

27. Byszewski, L, Lakshmikantham, V: Theorem about the existence and uniqueness of a solution of a nonlocal abstract Cauchy problem in a Banach space. Appl. Anal. 40, 11-19 (1991)

28. Hino, Y, Murakami, S, Naito, T: Functional-Differential Equations with Infinite Delay. Lecture Notes in Mathematics, vol. 1473. Springer, Berlin (1991)

29. Yan, BQ: Boundary value problems on the half-line with impulses and infinite delay. J. Math. Anal. Appl. 259, 94-114 (2001)

30. Kamenskii, M, Obukhovskii, V, Zecca, P: Condensing Multivalued Maps and Semilinear Differential Inclusions in Banach Spaces. de Gruyter, Berlin (2001)

31. Chadha, A, Pandey, DN: Existence and approximation of solution to neutral fractional differential equation with nonlocal conditions. Comput. Math. Appl. 69, 893-908 (2015)

32. Banas, J, Goebel, K: Measure of Noncompactness in Banach Spaces. Lecture Notes in Pure and Applied Mathematics. Marcel Dekker, New York (1980)

33. Agarwal, RP, Meehan, M, O'Regan, D: Fixed Point Theory and Applications. Cambridge Tracts in Mathematics, vol. 141. Cambridge University Press, Cambridge (2001)

34. Wang, JR, Zhou, Y, Fečkan, M: Alternative results and robustness for fractional evolution equations with periodic boundary conditions. Electron. J. Qual. Theory Differ. Equ. 2011, 97 (2011)

\section{Submit your manuscript to a SpringerOpen ${ }^{\circ}$ journal and benefit from:}

- Convenient online submission

- Rigorous peer review

- Immediate publication on acceptance

- Open access: articles freely available online

- High visibility within the field

Retaining the copyright to your article 